\title{
Uso de fármacos psicotrópicos por profissionais da saúde atuantes da área hospitalar
}

\author{
Use of psychotropic drugs by hospital health workers
}

Recebido em: 03/08/2014

Aceito em: $\quad 16 / 09 / 2014$
Ana Paula Helfer SCHNEIDER, Patricia Gens AZAMBUJA

Universidade de Santa Cruz do Sul - UNISC. Av. Independência, 2293.

Bairro Universitário, CEP: 96815-900. Santa Cruz do Sul, RS, Brasil. E-mail:

patriciagenz@yahoo.com.br

\section{ABSTRACT}

The aims of this study were to investigate the use of psychotropic drugs and factors associated with the use among health personnel at a hospital in Santa Cruz do Sul, RS factors. It was a cross-sectional study and the data collection was performed through a self-administered, unidentifiable and structured questionnaire with open and closed questions, between September and October 2012. 106 professionals were interviewed, $91.5 \%$ women, $74.5 \%$ nursing and $40.57 \%$ working in inpatient units. About the participants, 23 (21.7\%) were using psychotropics, with higher consumption, among professionals working in Intensive Care Units (34.78\%) as well as in inpatient units (34.78\%). These workers had over 60 hours weekly working (50\%), including cases of more than one bond employment. It could be observed a higher prevalence of the consumption of psychotropic medications among nursing staff (22.8\%). The main reasons for the early use of drugs studied were depression, anxiety and insomnia. The lack of knowledge about the characteristics of medicines and treatment were also observed. The results indicate a high use of psychotropic drugs among health professionals and that there is a need for more communication between prescribers and users, and the search for pharmaceutical care in order to clarify doubts regarding treatment, for proper rational use of medications.

Keywords: psychotropic drugs; health personnel. rational use of medications

\section{RESUMO}

Os objetivos deste estudo foram investigar a utilização de psicofármacos e os fatores associados ao seu uso entre os profissionais da saúde de um hospital de Santa Cruz do Sul, RS. Caracteriza-se por um estudo transversal, com coleta de dados por meio de questionário autoaplicável, não identificável e estruturado com perguntas abertas e fechadas, no período de setembro e outubro de 2012. Foram entrevistados 106 profissionais, sendo 91,5\% mulheres, 74,5\% técnicos de enfermagem e 40,57\% atuantes em Unidades de Internação. Dos 106 participantes da pesquisa, 23 (21,7\%) faziam uso de psicofármacos; com maior consumo entre profissionais atuantes em Unidades de Terapia Intensiva $(34,78 \%)$ e Unidades de Internação (34,78\%), com carga horária semanal de trabalho superior a 60 horas (50\%), incluindo situações de mais de um vínculo empregatício. Observou-se maior prevalência no consumo de psicofármacos entre técnicos de enfermagem (22,8\%). Os principais motivos apontados para o início do uso dos medicamentos em estudo foram a depressão, a ansiedade e a insônia. A falta de conhecimento sobre as características dos medicamentos e tratamento também foram observadas. Os resultados desse estudo apontam para um elevado consumo de psicofármacos entre profissionais de saúde e que há necessidade de maior comunicação entre usuários e prescritores, além da busca por orientação farmacêutica, a fim de elucidar dúvidas quanto ao tratamento, visando o uso racional e correto de medicamentos.

Palavras chave: psicofármacos; profissionais da saúde; uso racional de medicamentos 
INTRODUÇÃO

Os profissionais que lidam com pessoas doentes, especialmente no ambiente hospitalar, são propensos a se envolverem em situações de estresse, devido à complexidade das relações humanas e das tarefas, alta responsabilidade, estilo de vida irregular, além do desgaste físico e psíquico, inclusive por falta de apoio dos colegas e gestores $(1,2)$. Estudos têm demonstrado que a jornada de trabalho excessiva afeta a qualidade de vida dos profissionais, o que leva ao cansaço, redução da capacidade de trabalho e do raciocínio e desenvolvimento de distúrbios do sono e humor (3) além de apontarem alta prevalência de transtornos de ansiedade (42\%) e depressão $(22 \%)$ entre diversos profissionais, relacionados às atividades laborais (4).

Em estudo realizado em 2003, com métodos padronizados internacionalmente pela World Health Survey, $18,8 \%$ dos brasileiros relataram ter recebido um diagnóstico de depressão nos últimos 12 meses. Entretanto, um estudo com critérios mais amplos para transtornos mentais comuns (TMC; estados mistos de depressão e ansiedade) relatou que cerca de $30 \%$ dos adultos brasileiros apresentaram tais sintomas (5). Em estudo realizado com trabalhadores da enfermagem, a prevalência de transtorno mental comum (TMC) foi de 35,0\% (6). Com a alta prevalência de depressão e TCM, tem crescido a utilização de psicofármacos entre a população e entre trabalhadores da saúde (7).

$\mathrm{O}$ estresse resultante do desgaste físico e/ou emocional no meio ocupacional ou insatisfação com a qualidade de vida pode acarretar em prestação de serviços inadequada, fator especialmente preocupante quando referente a profissionais da saúde. Pesquisas apontam o trabalho de profissionais de saúde como altamente estressante, com afastamentos do trabalho por parte da equipe de enfermagem devido a transtornos psicológicos (8); elevada prevalência de síndrome de burnout entre médicos (9), sendo essa síndrome considerada fator de risco mais importante para o desenvolvimento de ansiedade e insônia como observado por Sun e cols (2012) (10). A síndrome de burnout, ou síndrome do esgotamento profissional, é uma manifestação essencialmente social que reflete os sentimentos de crise e desorientação encontrados na sociedade moderna. É causada pelo estresse crônico, tipicamente observado em ambientes de trabalho, especialmente quando ocorre pressão excessiva, e os conflitos estão presentes, e quando há uma escassez de recompensas emocionais, reconhecimento e sucesso, principalmente observado nos trabalhadores da saúde (11).
Neste contexto, os transtornos psicológicos e o estresse ocupacional têm levado muitos profissionais à procura por psicofármacos para o alívio de seus problemas; porém, se os fatores estressantes continuam presentes na vida do indivíduo, é provável que este faça uso irracional do medicamento, podendo acarretar riscos à sua saúde $(12,13)$.

Assim, o presente estudo teve por objetivo avaliar o consumo de psicofármacos por profissionais da saúde da área hospitalar e os fatores sócio-demográficos, profissionais e comportamentais associados ao seu uso.

\section{MÉTODO}

Foi realizado um estudo analítico observacional transversal com abordagem quantitativa em hospital da região central do Rio Grande do Sul, entre os meses de setembro e outubro de 2012. Foram convidados a participar da pesquisa profissionais da saúde de ensino superior e técnico, atuantes no hospital, maiores de 18 anos e que assinaram o Termo de Consentimento Livre e Esclarecido (TCLE). A amostra total foi de 106 profissionais, selecionados aleatoriamente, dos turnos diurno e noturno, entre eles: técnicos de enfermagem, médicos, enfermeiros, farmacêuticos, nutricionistas, psicólogas e fisioterapeutas.

Esta pesquisa foi aprovada pelo Comitê de Ética em Pesquisa da Universidade de Santa Cruz do Sul sob o número de protocolo $\mathrm{n}^{\circ} 88.279 / 2012$. Os profissionais de saúde foram consultados sobre a participação voluntária no projeto e aqueles que aceitaram participar assinaram o TCLE e preencheram um questionário autoaplicável e não identificável, formulado com 28 questões abertas e fechadas. Os participantes foram informados quanto à finalidade do estudo, sobre o sigilo quanto à identidade e sobre o direito de suspender a participação a qualquer momento. $\mathrm{O}$ questionário foi entregue em um envelope aos participantes da pesquisa, que poderiam esclarecer dúvidas diretamente com a pesquisadora, que se mantinha presente durante o preenchimento. Após esta etapa, os questionários foram depositados pelos participantes junto aos envelopes dentro de uma urna. Aqueles que se encontravam ocupados puderam entregar o questionário em outro momento.

O questionário continha quatro partes; na primeira, foram coletados dados relacionados à caracterização dos trabalhadores, como variáveis sócio-demográficas (sexo, idade, estado civil); e dados profissionais (profissão, setor, carga de trabalho semanal, turno, realização ou não de plantão noturno, trabalho ou não aos finais de semana, tempo de trabalho no hospital, existência 
ou não de outra atividade profissional além da desempenhada no hospital). Na segunda parte havia questões que avaliavam o uso dos psicofármacos (se fazia ou não uso), motivo do uso, qual/quais, se apresentava doença concomitante, tempo de uso); na terceira parte foi avaliado o conhecimento dos usuários quanto aos psicofármacos e ao tratamento (se havia aumentado a dose sem o conhecimento do médico, período para o início dos efeitos do medicamento, se o medicamento causa ou não efeitos colaterais, tempo que deverá fazer uso do mesmo, se o medicamento pode causar dependência ou tolerância, como deve ser feita a remoção do fármaco) e ao final dessa parte foi perguntado ao participante, se o mesmo tinha dúvidas quanto ao tratamento; na quarta parte foram coletadas informações sobre variáveis comportamentais (consumo ou não de bebida alcoólica, tabaco, prática ou não de atividade física, refeições irregulares ou não).

Os dados foram digitados no Microsoft Excel $^{\circledR}$ e analisados no software SPSS versão 18.0 (Statistical Package for the Social Sciences 18.0). Foram realizadas análises descritivas, univariadas, aplicados os testes qui-quadrado de Pearson e associação linear, sendo considerado como desfecho principal o uso de psicofármacos.

\section{RESULTADOS E DISCUSSÃO}

Dos 106 profissionais de saúde que participaram da pesquisa, $75,5 \%$ trabalhavam no turno diurno e $24,5 \%$ no noturno. Entre os entrevistados, $91,5 \%$ eram do sexo feminino; a faixa etária predominante foi 18 a 29 anos (47,2\%). Quanto ao estado civil, a maioria dos participantes possuía companheiro/a (49,1\%). Em relação aos dados profissionais, a profissão de maior participação na pesquisa foi a de técnico em enfermagem $(74,5 \%)$, seguida de enfermeiros $(11,3 \%)$. Os setores mais participativos foram as Unidades de Internação de adultos (40,6\%); 56,6\% dos participantes trabalhavam no hospital por período menor ou igual a 4 anos; $85,8 \%$ da amostra trabalhava de 21 a 40 horas semanais, sendo $75,5 \%$ durante o dia; e 78,3\% afirmaram trabalhar às vezes aos finais de semana. A maior parte não realizava plantão noturno $(67,9 \%)$ e não desempenhava outra atividade profissional $(65,1 \%)$.

A Tabela 1 apresenta a prevalência do uso de fármacos psicotrópicos e as características sociodemográficas, profissionais e comportamentais dos participantes do estudo.
Quando questionados sobre atividade física, 58,5\% responderam não praticar com frequência; quanto às refeições, $63,2 \%$ afirmaram realizar refeições de forma irregular; e quanto ao uso de tabaco e de álcool, $96,2 \% \mathrm{e}$ $67 \%$, respectivamente, relataram não consumi-los.

A prevalência de uso de psicofármacos foi $21,7 \%$ (22,2\% nos homens e $21,6 \%$ nas mulheres). O uso de psicofármacos esteve associado com a faixa etária e profissão, com prevalência de $100 \%$ nos participantes com idade superior a 50 anos, seguido de $27,3 \%$ nos profissionais com faixa etária entre 30 a 39 anos $(\mathrm{p}<0,05)$. Também houve alta prevalência do uso de psicofármacos por nutricionistas $(100 \%)$ e técnicos de enfermagem $(22,8 \%)(\mathrm{p}<0,05)$.

Apesar de não apresentarem associação significativa, destaca-se a maior prevalência de uso de psicofármacos entre os usuários com companheiro/a (26,9\%), os que trabalhavam nas Unidades de Internação e UTI (34,78\% cada), os indivíduos com carga horária superior a 60 horas semanais (50\%), os trabalhadores que desempenhavam atividade profissional aos finais de semana $(22,9 \%)$ e entre os indivíduos que não desempenhavam outra atividade profissional $(65,7 \%)$.

Em relação às variáveis comportamentais, houve maior prevalência do uso de psicofármacos em $28,2 \%$ daqueles que afirmaram não realizar refeições regulares; em $22,6 \%$ dos participantes que não praticavam atividade física com frequência; em 22,5\% que não fumavam e em $25,4 \%$ que não consumiam bebida alcoólica.

A Tabela 2 apresenta os transtornos de maior frequência e o conhecimento dos usuários quanto aos medicamentos e tratamento. O transtorno de maior prevalência foi depressão $(30,43 \%)$, seguido de ansiedade $(17,39 \%)$ e insônia $(13,04 \%) ; 21,75 \%$ dos indivíduos faziam uso do psicofármaco pelo período de 1 a 2 anos; $82,6 \%$ revelaram nunca ter aumentado a dose sem consultar o médico; $52 \%$ dos usuários interromperam o tratamento sem consultar o médico. Quando questionados sobre o tempo que o medicamento levaria para fazer efeito: $39,1 \%$ responderam que levaria 2 semanas ou mais; $73,9 \%$ dos usuários responderam que o medicamento estava causando efeito colateral; $65 \%$ não sabiam por quanto tempo deveria manter o tratamento; $47,8 \%$ e $26,1 \%$ responderam que o medicamento não causava dependência, nem tolerância, respectivamente; $65,2 \%$ responderam que a remoção deveria ser de forma gradual e $74 \%$ que não tinham dúvidas quanto ao tratamento. 
Tabela 1: Variáveis sociodemográficas, profissionais, comportamentais e de saúde de profissionais de saúde de hospital do Rio Grande do Sul, Brasil, no período de setembro e outubro de 2012

\begin{tabular}{|c|c|c|c|c|}
\hline Variável & $\mathbf{N}$ & Percentual (\%) & Prevalência(\%) & Valor-p \\
\hline \multicolumn{5}{|l|}{ Sexo } \\
\hline Masculino & 9 & 8,5 & 22,2 & 0,968 \\
\hline Feminino & 97 & 91,5 & 21,6 & \\
\hline \multicolumn{5}{|l|}{ Faixa Etária } \\
\hline 18 a 29 anos & 50 & 47,2 & 18,0 & $<0,05$ \\
\hline 30 a 39 anos & 33 & 31,1 & 27,3 & \\
\hline 40 a 49 anos & 13 & 12,3 & 7,7 & \\
\hline $\begin{array}{l}\text { > } 50 \text { anos } \\
\text { *Abstenção }\end{array}$ & $\begin{array}{l}2 \\
8\end{array}$ & $\begin{array}{l}1,9 \\
7,5\end{array}$ & 100 & \\
\hline \multicolumn{5}{|l|}{ Profissão } \\
\hline Enfermeiro/a & 12 & 11,3 & 8,3 & $<0,05$ \\
\hline Farmacêutico/a & 2 & 1,9 & 0 & \\
\hline Fisioterapeuta & 3 & 2,8 & 0 & \\
\hline Médico/a & 5 & 4,8 & 20 & \\
\hline Nutricionista & 3 & 2,8 & 100 & \\
\hline Psicólogo/a & 2 & 1,9 & 0 & \\
\hline Técnico/a em enfermagem & 79 & 74,5 & 22,8 & \\
\hline \multicolumn{5}{|l|}{ Estado Civil } \\
\hline Solteiro/a & 44 & 41,5 & 15,9 & 0,278 \\
\hline Com companheiro/a & 52 & 49,1 & 26,9 & \\
\hline $\begin{array}{l}\text { Separado/a ou viúvo/a } \\
\text { * Abstenção }\end{array}$ & $\begin{array}{l}3 \\
7\end{array}$ & $\begin{array}{l}2,8 \\
6,6\end{array}$ & 0 & \\
\hline \multicolumn{5}{|l|}{ Tempo de trabalho no hospital } \\
\hline$<1$ ano & 28 & 26,4 & 35,0 & 0,589 \\
\hline 1 a 4 anos & 32 & 30,2 & 40,0 & \\
\hline 5 a 9 anos & 9 & 8,5 & 11,2 & \\
\hline$>10$ anos & 11 & 10,4 & 13,8 & \\
\hline *Abstenção & 26 & 24,5 & & \\
\hline \multicolumn{5}{|l|}{ Setor } \\
\hline Unidade de Internação & 43 & 40,6 & 34,8 & 0,601 \\
\hline Unidade Tratamento Intensivo & 27 & 25,5 & 34,8 & \\
\hline Bloco Cirúrgico & 9 & 8,5 & 13,0 & \\
\hline Centro Obstétrico & 5 & 4,7 & 0 & \\
\hline Emergência & 12 & 11,3 & 4,4 & \\
\hline Outros & 10 & 9,4 & 13,0 & \\
\hline \multicolumn{5}{|l|}{ Carga horária } \\
\hline Até 20h & 3 & 2,8 & 0 & 0,423 \\
\hline 21 a $40 h$ & 91 & 85,8 & 20,9 & \\
\hline 41 a $60 h$ & 8 & 7,6 & 25,0 & \\
\hline$>60 \mathrm{~h}$ & 4 & 3,8 & 50,0 & \\
\hline \multicolumn{5}{|l|}{ Turno } \\
\hline Diurno & 80 & 75,5 & 25,0 & 0,220 \\
\hline Noturno & 26 & 24,5 & 11,5 & \\
\hline \multicolumn{5}{|l|}{ Trabalha nos finais de semana } \\
\hline Sempre & 20 & 18,9 & 22,9 & 0,627 \\
\hline As vezes & 83 & 78,3 & 20,0 & \\
\hline Nunca & 3 & 2,8 & 0 & \\
\hline
\end{tabular}




\begin{tabular}{|l|r|r|r|c|}
\hline \multicolumn{1}{|c|}{ Variável } & N & Percentual (\%) & Prevalência(\%) & Valor-p \\
\hline Outra atividade profissional & & & & 34,3 \\
\hline Sim & 36 & 34,0 & 65,7 & 0,868 \\
\hline Não & 69 & 65,1 & & \\
\hline *Abstenção & 1 & 0,9 & \\
\hline
\end{tabular}

*Participantes da pesquisa que não preencheram a questão.

No presente estudo, a prevalência de uso de psicofármacos entre profissionais de saúde da área hospitalar $(21,7 \%)$ foi superior ao estudo de base populacional realizado em São Paulo, com 2000 adultos, onde a prevalência de uso de psicofármacos foi de 15,8\% (14). Estudos apontam que profissionais que realizam trabalho de alta demanda e baixo controle (alta exigência) apresentam alta prevalência de transtornos mentais comuns $(15,16)$, o que pode resultar na utilização de psicofármacos. Em um estudo transversal, com 309 profissionais de enfermagem que trabalhavam em atividades assistenciais em um hospital de grande porte em Feira de Santa, BA, foi observado que, dentre os problemas de saúde, destacaram-se as queixas e diagnósticos de problemas mentais e posturais, que podem estar associados às características do trabalho de enfermagem: atender a pacientes, enfrentar a dor, o sofrimento a morte, excesso de trabalho, elevada responsabilidade, atividades de plantão e baixa remuneração (6). Assim, a existência nos trabalhadores da saúde de condições desencadeadoras de sofrimento, estresse e ansiedade, é uma realidade não mais contestada $(8,15,17-20)$. No entanto, as repercussões sobre a saúde desses trabalhadores, reconhecidamente expostos a fatores estressantes e ansiogênicos, ainda são pouco conhecidas e estudadas (6). No presente estudo, foi observada maior prevalência do uso de psicofármacos entre nutricionistas $(100 \%)$ e técnicos de enfermagem $(22,8 \%)(\mathrm{p}<0,05)$.

Outro ponto onde foi verificada a prevalência de uso de psicofármacos foi entre os profissionais atuantes nos setores de Unidades de Internação $(34,78 \%)$ e Unidades de Terapia Intensiva (UTI) (34,78\%). Nas UTI, os profissionais têm maior proximidade com os pacientes, devido à gravidade do estado de saúde, o que consome mais tempo para o desempenho das atividades, pelo alto nível de dependência (21). Esses fatores, associados ao estado de alerta para realização de um rápido atendimento quando necessário podem desencadear estresse. No estudo de Barros e cols. (2008), médicos atuantes na UTI apontaram alguns fatores como estressantes, como ruídos excessivos no setor, possíveis complicações no atendimento aos pacientes, lidar com o sofrimento e morte e com diversas atividades simultaneamente e em ritmo acelerado, problemas administrativos, pressão para dar alta aos pacientes e quantidade excessiva de pacientes sob a responsabilidade de cada profissional (9).

Foi verificado que a maioria dos profissionais não desempenhava outra atividade profissional $(65,1 \%)$. Este dado difere dos resultados encontrados por Rios e cols. (2010), os quais mostraram que $52 \%$ dos participantes da pesquisa tinham mais de dois empregos (22). Porém, o presente estudo assemelha-se com o de Paschoa e cols. (2007), onde $67,5 \%$ das entrevistadas não dedicavam-se a outra atividade profissional (23). A maior parte da amostra $(67,9 \%)$ relatou não realizar plantão noturno, mas apresentou maior prevalência $(25 \%)$ do uso de fármacos psicotrópicos. Com base nesses dados, a dupla jornada de trabalho e o plantão noturno, fatores associados ao estresse ocupacional (1), não foram considerados como agentes potenciais para o desenvolvimento de transtornos no presente estudo.

Dentre os principais motivos que levaram os profissionais de saúde a utilizar psicofármacos estão: a depressão $(30,4 \%)$, ansiedade $(17,4 \%)$ e insônia $(13,0 \%)$. Em outro levantamento, os principais motivos que levaram os pacientes a procurar os serviços de uma unidade de apoio psicossocial, entre 2005 e 2008, foram transtorno de ansiedade (42\%) e depressão (22\%) (4). Uma pesquisa de Carreiro (2010) sobre o impacto do trabalho na saúde mental de profissionais da Estratégia de Saúde da Família (ESF), apontou que 86,3\% dos entrevistados referiam sofrer com distúrbios de ansiedade e 13,7\% de depressão (19).

Quanto ao conhecimento sobre a ação dos psicofármacos, como a maioria dos usuários marcou a opção "duas semanas ou mais" e, como a maior parte da amostra afirmou sofrer depressão, pode-se inferir que sabiam qual o tempo para início de ação dos mesmos. A literatura mostra que os antidepressivos tricíclicos, inibidores seletivos da recaptação da serotonina e inibidores da monoaminoxidase tem início de ação após 2 ou 3 semanas de tratamento (19).

Grande parte dos usuários afirmou fazer uso de medicamento há 1 ou 2 anos; que o mesmo estava causando efeitos colaterais; que já havia interrompido o tratamento sem consultar o médico; e que não sabia por quanto tempo deveria permanecer em 
tratamento. Pode haver relação entre dúvidas quanto ao tratamento, presença de efeitos colaterais e interrupção do tratamento sem consentimento médico. Um dos principais motivos da não-adesão ao tratamento com psicofármacos são os efeitos colaterais, e para reverter esse quadro, é importante a informação acerca daqueles mais frequentes e o tempo que eles podem persistir até que o medicamento comece a surtir o efeito terapêutico desejado $(24,25)$.

A amostra pretendida era de 150 profissionais, porém, houve limitações no presente estudo que devem ser mencionadas.

Tabela 2: Prevalência do uso de psicofármacos por profissionais de saúde de hospital do Rio Grande do Sul, Brasil, no período de setembro e outubro de 2012

\begin{tabular}{|c|c|c|}
\hline Variável & $\mathbf{N}$ & Percentual (\%) \\
\hline \multicolumn{3}{|l|}{ Motivo do uso de psicofármacos } \\
\hline Depressão & 7 & 30,4 \\
\hline Ansiedade & 3 & 17,4 \\
\hline Insônia & 3 & 13,0 \\
\hline Depressão, ansiedade e insônia & 2 & 8,7 \\
\hline Depressão, insônia, síndrome do pânico & 1 & 4,3 \\
\hline Ansiedade, insônia & 2 & 8,7 \\
\hline Insônia, transtorno de déficit de atenção/hiperatividade & 1 & 4,3 \\
\hline Insônia, enxaqueca & 1 & 4,3 \\
\hline Obesidade & 1 & 4,3 \\
\hline Dor intensa & 1 & 4,3 \\
\hline \multicolumn{3}{|l|}{ Tempo de uso } \\
\hline$<1$ mês & 3 & 13,0 \\
\hline 1 a 5 meses & 4 & 17,4 \\
\hline 6 a 11 meses & 4 & 17,4 \\
\hline 1 a 2 anos & 5 & 21,7 \\
\hline 3 a 4 anos & 4 & 17,4 \\
\hline$>5$ anos & 3 & 13,0 \\
\hline \multicolumn{3}{|l|}{ Já aumentou a dose sem consultar o médico } \\
\hline Nunca & 19 & 82,6 \\
\hline Uma vez & 2 & 8,7 \\
\hline Frequentemente & 2 & 8,7 \\
\hline \multicolumn{3}{|l|}{ Interrompeu o tratamento sem consultar o médico } \\
\hline $\operatorname{Sim}$ & 12 & 52 \\
\hline Não & 11 & 48 \\
\hline \multicolumn{3}{|l|}{ Início de ação terapêutica do fármaco } \\
\hline Até $1 \mathrm{~h}$ & 4 & 17,4 \\
\hline $12 \mathrm{~h}$ & 4 & 17,4 \\
\hline 1 dia & 2 & 8,7 \\
\hline 2 semanas ou mais & 9 & 39,1 \\
\hline Não sabe & 4 & 17,4 \\
\hline \multicolumn{3}{|l|}{0 fármaco está causando efeito colateral } \\
\hline Sim & 17 & 73,9 \\
\hline Não & 4 & 17,4 \\
\hline Não sabe & 2 & 8,7 \\
\hline \multicolumn{3}{|l|}{ Sabe quanto tempo deverá usar o medicamento } \\
\hline Sim & 8 & 35 \\
\hline Não & 15 & 65 \\
\hline \multicolumn{3}{|l|}{0 psicofármaco pode causar dependência } \\
\hline $\operatorname{Sim}$ & 10 & 43,5 \\
\hline Não & 11 & 47,8 \\
\hline Não sabe & 2 & 8,7 \\
\hline
\end{tabular}




\begin{tabular}{|c|c|c|}
\hline $\begin{array}{lll}\text { Variável } \\
\end{array}$ & N & Percentual (\%) \\
\hline \multicolumn{3}{|l|}{0 psicofármaco pode causar tolerância } \\
\hline Sim & 9 & 39,1 \\
\hline Não & 6 & 26,1 \\
\hline Não sabe & 8 & 34,8 \\
\hline \multicolumn{3}{|c|}{0 psicofármaco deve ser removido de forma gradual } \\
\hline Sim & 15 & 65,2 \\
\hline Não & 4 & 17,4 \\
\hline Não sabe & 4 & 17,4 \\
\hline \multicolumn{3}{|l|}{ Tem dúvidas quanto ao tratamento } \\
\hline Sim & 6 & 26 \\
\hline Não & 17 & 74 \\
\hline
\end{tabular}

Alguns profissionais não aceitaram participar da pesquisa e a maioria não pôde preencher o questionário no momento da abordagem devido às atividades profissionais.Nesse caso, os questionários foram deixados com os participantes ou com o responsável do setor e foi marcado outro horário ou dia para a retirada. Diante deste fato, algumas pessoas que aceitaram participar da pesquisa levaram o questionário para casa e não devolveram no dia marcado. Como o questionário era autoaplicável, algumas perguntas não foram respondidas. Houve pouca participação de médicos, pois estes passam boa parte do horário de trabalho em atendimento aos pacientes e muitos atuam em diversos setores do hospital e ainda desempenham atividade profissional em consultório; diferente de enfermeiros e técnicos de enfermagem, que encontram-se com frequência nos postos de enfermagem, devido a questões administrativas e retirada de medicamentos e materiais para realização dos procedimentos e atuam em setores específicos.

Não é incomum, nos dias de hoje, discriminação contra indivíduos que fazem uso de psicofármacos, e por esse motivo, muitos deles não admitem utilizá-los; inclusive, em alguns casos, não aderindo ao tratamento por preconceito. Os psicofármacos somente devem ser utilizados após diagnóstico e orientação médica. Quan- do necessários, são muito úteis no tratamento de doenças que atingem o sistema nervoso central (SNC), porém, como todos os fármacos, apresentam efeitos colaterais e graves quando utilizados de maneira inadequada, podendo levar à intoxicação e até mesmo à morte. Por isso, é muito importante que os usuários recebam todas as informações necessárias sobre o tratamento, evitando que ocorra o uso irracional do medicamento.

\section{RESULTADOS:}

Foi observado que os profissionais não detêm a informação necessária para o uso correto do medicamento; consequentemente, interferindo no sucesso do tratamento. Para que haja adesão ao tratamento, é necessário que o usuário tenha conhecimento sobre seu problema de saúde, o medicamento em uso e o tratamento, e para que isso aconteça é necessária maior comunicação entre ele, os profissionais prescritores, o farmacêutico e outros que se fizerem necessários dentro da equipe multidisciplinar. Este tipo de estudo possibilita o desenvolvimento de estratégias para promover o uso adequado dos medicamentos, uma vez que a não adesão ou o uso inadequado representam risco à saúde do trabalhador e refletem de forma negativa no atendimento dentro do ambiente institucional.

\section{REFERÊNCIAS}

1. Schmidt DRC, Dantas RAS, Marziale MHP, Laus AM. Estresse ocupacional entre profissionais de enfermagem do bloco cirúrgico. Texto \& Contexto - Enfermagem. 2009;18:330-7.

2. Ebling M, Carlotto MS. Burnout syndrome and associated factors among health professionals of a public hospital. Trends Psychiatry Psychother. 2012; 34:93-100.
3. Neto GD. Stress e fadiga na segurança do ato anestésico: impacto no desempenho profissional. Medicina Perioperatória, SAERJ, Rio de Janeiro. 2006:967-71.

4. Sales A, Lemos I, Aguiar A, Caldas M. O cuidador cuidado: a experiência de cuidar de quem cuida. Neurobiol. 2010;73(1):61-73. 
5. Schmidt MI, Duncan BB, e Silva GA, Menezes AM, Monteiro CA, Barreto SM, et al. Chronic non-communicable diseases in Brazil: burden and current challenges. The Lancet. 2011; 377(9781):1949-1961.

6. Rodrigues EP, Rodrigues US, Oliveira LdMM, Laudano RCS, Sobrinho CLN. Prevalência de transtornos mentais comuns em trabalhadores de enfermagem em um hospital da Bahia. Revista Brasileira de Enfermagem. 2014; 67:296-301.

7. Rodrigues MAP, Facchini LA, Lima MSd. Modificações nos padrões de consumo de psicofármacos em localidade do Sul do Brasil. Revista de Saúde Pública. 2006;40:107114.

8. de Faria AC, Barboza DB, Domingos NA. Absenteísmo por transtornos mentais na enfermagem no período de 1995 a 2004. Arq Ciênc Saúde 2005; 12(1): 14-20.

9. Barros DS, Tironi MOS, Sobrinho CLN, Neves FS, Bitencourt AGV, Almeida AM, et al. Médicos plantonistas de unidade de terapia intensiva: perfil sócio-demográfico, condições de trabalho e fatores associados à síndrome de burnout. Rev Bras Ter Intens. 2010; 20(3):235-40.

10. Sun W, Fu J, Chang Y, Wang L. Epidemiological study on risk factors for anxiety disorder among Chinese doctors. Journal of occupational health. 2012;54(1):1-8.

11. Gil-Monte PR. El síndrome de quemarse por el trabajo (Burnout): una enfermedad laboral en la sociedad del bienestar: Ed Pirámide; 2005.

12. Díaz L, Ulloa CM, Taubert De Freitas F, Amorim L, Barcelos MC, Valenzuela SV, et al. El uso de drogas en el personal de enfermería. Ciencia y enfermería. 2011; 17(2):37-45.

13. Pelegrini MRF. O abuso de medicamentos psicotrópicos na contemporaneidade. Psicologia: ciência e profissão. 2003; 23(1):38-41.

14. Blay SL, Fillenbaum GG, Pitta JC, Peluso ET. Factors associated with antidepressant, anxiolytic, and other psychotropic medication use to treat psychiatric symptoms in the city of Sao Paulo, Brazil. Int Clin Psychopharmacol. 2014 May;29(3):157-165. DOI:10.1097/YIC. 0000000000000008
15. Araújo TM, Aquino E, Menezes G, Santos CO, Aguiar L Aspectos psicossociais do trabalho e distúrbios psíquicos entre trabalhadoras de enfermagem. Rev Saúde Pública. 2003;37:424-433.

16. World Health Organization. Neuroscience of psychoactive substance use and dependence 2004 [Acesso em: 28/08/2014]. Available from: http://www.who.int/ substance_abuse/publications/en/Neuroscience.pdf.

17. Silva AA, Souza JMP, Borges FNS, Fischer FM. Healthrelated quality of life and working conditions among nursing providers. Rev Saúde Pública. 2010; 44:718-725.

18. Barbosa GB, Correia AKS, Oliveira LMM, Santos VC, Ferreira SMS, Martins Júnior DF, et al. Trabalho e saúde mental dos profissionais da Estratégia Saúde da Família em um município do Estado da Bahia, Brasil. Rev Bras Saúde Ocup. 2012; 37:306-315.

19. Carreiro GSP. O impacto do trabalho na saúde mental dos profissionais da estratégia saúde da família. [Dissertação]. João Pessoa - Paraíba: Universidade Federal da Paraíba; 2010.

20. Braga LC, Carvalho LR, Binder MCP. Condições de trabalho e transtornos mentais comuns em trabalhadores da rede básica de saúde de Botucatu (SP). Ciência \& Saúde Coletiva. 2010; 15:1585-1596.

21. Elias MA, Navarro VL. A relação entre o trabalho, a saúde e as condições de vida: negatividade e positividade no trabalho das profissionais de enfermagem de um hospital escola. Rev Lat Am Enfermagem. 2006; 14(3):517-525.

22. Rios KA, Barbosa DA, Belasco AGS. Avaliação de qualidade de vida e depressão de técnicos e auxiliares de enfermagem. Rev Lat Am Enfermagem. 2010;18(3):413-20.

23. Paschoa S, Zanei SSV, Whitaker IY. Qualidade de vida dos trabalhadores de enfermagem de unidades de terapia intensiva. Acta Paul Enferm. 2007;20(3):305-10.

24. Marangell LB, Silver JM, Martinez JM, Yudofsky SC. Psicofarmacologia: Artmed; 2003.

25. Brunton LL, Chabner BA, Knollmann BC. As bases farmacológicas da terapêutica de Goodman \& Gilman: McGraw Hill Brasil; 2006. 